Conclusions This study provides evidence for increasing admission to first rehabilitation of the elderly for TSCIs, which can in part be attributed to an increase in risk of TSCI with increasing age. Potential lifestyle changes among the youngest, especially for sports and leisure-related TSCIs, as well as the oldest, possibly due to increase in activity, could contribute to the observed increase in risk.

\section{IMPLEMENTATION AND EVALUATION OF AN MHEALTH SYSTEM FOR ACUTE BURN INJURIES IN RESOURCE- CONSTRAINED SETTINGS}

${ }^{1}$ Marie Hasselberg, ${ }^{2}$ Lee Wallis, ${ }^{3}$ Hendry Sawe, 'Lucie Laflamme. 'Karolinska Institutet, Sweden; ${ }^{2}$ Stellenbosch University; ${ }^{3}$ Muhimbile University

\subsection{6/injuryprev-2016-042156.309}

Background Burn injuries are a leading cause of premature death worldwide and are largely attributable to poor living conditions. Timely care is a prerequisite to reduce morbidity and mortality and it can be significantly improved by smartphone-based consultation systems. The overall aim of this project is to assess how an mHealth system for acute burn injury care can impact on the delivery of emergency care for burns victims in terms of clinical management.

Methods The clinical quality includes assessments of diagnostic accuracy and patient management. Bedside diagnosis or image based diagnosis by burns experts will be used as gold standard. Technology Usability Evaluation Model will be used to assess how the users experience the system.

Results A tele-consultation system for acute burn care is currently under implementation in the Western Cape, South Africa. An application (app) is installed on a smartphone located in each of the emergency services of 8 hospitals to transmit visual and textual information between emergency staff at point of care and a network of burns specialists. A stepwise implementation of the system will start in Tanzania in the beginning of 2016. The burn specialists already involved in South Africa will act as experts for Tanzania. The evaluation will build on an evaluation already started in South Africa. The first part of the evaluation will include the hospitals currently using the mHealth system in the Western Cape, South Africa and 2-3 hospitals in Tanzania. The mHealth system and results of the evaluation will be presented at the conference.

Conclusions Low cost and timely alternatives to burn injury control are a pressing need in many low- and middle-income settings and countries. This project is a determinant step in that direction and can lead to the implementation of a viable, inclusive, and environment friendly mHealth system.

\section{COMMON MORTALITY MODELS FAIL TO USE ANATOMIC INJURY INFORMATION OPTIMALLY}

${ }^{1}$ Alan Cook, ${ }^{2}$ Turner Osler, ${ }^{3}$ Laurent Glance, ${ }^{4}$ Fiona Lecky, ${ }^{5}$ Omar Bouamra. ${ }^{1}$ Chandler Regional Medical Centre, USA; ${ }^{2}$ University of Vermont, USA; ${ }^{3}$ University of Rochester, USA; ${ }^{4}$ University of Sheffield, UK; ${ }^{5}$ University of Manchester, UK

\subsection{6/injuryprev-2016-042156.310}

Background Comparison of trauma centres requires accurate injury severity metrics. The Trauma Audit and Research Network (TARN) in the UK, and the Trauma Injury Severity Score (TRISS) provide such risk adjustment. These models incorporate the Injury Severity Score (ISS) as the measure of anatomic injury severity but categorise it differently. The Trauma Mortality
Prediction Model (TMPM) better predicts mortality than the ISS. We compared the anatomic injury components of TARN, and TRISS models to TMPM.

Methods Data from the National Trauma Data Bank for 20022012. Probability of death was estimated for TARN, and TRISS from ISS values according to each model's treatment and compared these to TMPM using measures of discrimination (area under ROC curves), proximity to the true model (Akaike Information Criterion (AIC)), and calibration (Hosmer-Lemeshow statistic (HL)). Confidence intervals (CI) were estimated by bootstrapping. ISS and TMPM values were estimated for the Abbreviated Injury Scale (AIS) and the International Classification of Diseases, $9^{\text {th }}$ Revision (ICD-9) lexicons. Data are shown as medians and 95\% CI.

Results $\mathrm{N}=1,145,959$

\begin{tabular}{lllllll}
\hline AIS & ROC & \multicolumn{3}{l}{ AIC } & HL & \\
\hline TMPM & 0.8716 & $0.8695-0.8734$ & 238130 & $236110-240201$ & 226.5 & $168.5-289.7$ \\
TARN & 0.8374 & $0.8351-0.8396$ & 270017 & $267958-272213$ & 668.5 & $586.0-745.4$ \\
TRISS & 0.8368 & $0.8346-0.8389$ & 275098 & $273058-277265$ & 2335.3 & $2186.9-2516.4$ \\
ICD-9 & ROC & & AIC & & HL & \\
TMPM & 0.8475 & $0.8451-0.8498$ & 211424 & $209531-213377$ & 107.1 & $76.1-142.3$ \\
TRISS & 0.8234 & $0.8211-0.8258$ & 223081 & $221132-225128$ & 1850.0 & $1702.9-1998.7$ \\
TARN & 0.8131 & $0.8106-0.8153$ & 227002 & $225115-228955$ & 1033.1 & $929.0-1152.4$ \\
\hline
\end{tabular}

Conclusions TMPM better predicted mortality than did the anatomic injury component of TRISS, or TARN and this was true whether individual injuries were coded in the AIS lexicon or in the ICD-9. As trauma care evolves, so must models of mortality prediction that best capture injury severity.

\section{Injury Statistics}

\section{Parallel Tue 2.6}

\section{APPLICATION OF SPATIAL EPIDEMIOLOGICAL APPROACHES TO INJURY RESEARCH: A SYSTEMATIC REVIEW}

Himalaya Singh, Lauren V Fortington, Caroline Finch. Federation University Australia

\subsection{6/injuryprev-2016-042156.311}

Background Injuries are a leading cause of death and disability around the world. Previous studies have shown that certain populations are consistently at greater risk of injury. Spatial epidemiological approach provides a way to better understand injury patterns and their associated risk factors at a population level. The aim of this research is to provide a systematic reivew of spatial epidemiological methods applied to injury research.

Methods A search was conducted in three major electronic databases (PubMed, Web of Science and Science Direct), for papers published between 2000-2015 inclusive. Included were papers reporting unintentional injury outcomes, which used geospatial methods for spatial epidemiological analysis. Findings are reported using PRISMA (Preferred Reporting Items for Systematic Reviews and Meta-Analyses) guidelines.

Results From over 10,000 articles, 88 articles met all inclusion criteria. The major categories of injury data that have been reported with geospatial methods were road traffic (52\%), falls $(11 \%)$, burns $(12 \%)$, drowning $(5 \%)$, workplace injuries $(2 \%)$ 